\title{
Guest editorial for special issue on massively multiplayer online gaming systems and applications
}

\author{
Shervin Shirmohammadi • Mark Claypool
}

Published online: 12 June 2009

(C) Springer Science + Business Media, LLC 2009

Massively Multiplayer Online Games (MMOGs), now a profitable sector of the industry and subject to academic and industry research, are gaming platforms that allow hundreds of thousands of players to simultaneously play in the same online game, often interacting with tens or even hundreds of other players. Although originally designed for gaming, MMOGs are now widely used for socializing, business, commerce, scientific experimentation, and many other practical purposes. One could say that MMOGs are the "killer app" that brings Virtual Reality into the realm of eSociety. This is evident from the fact that real companies are opening "virtual branches" in these online games, such as Reebok, IBM, and CNN, to name a few. Virtual currencies such as Linden (or L\$) in Second Life are already being exchanged for real-world money ${ }^{1}$. Similarly, virtual goods and virtual real-state are being bought and sold with real-world money. Massive numbers of users spend their time with their fellow players at online games like EverQuest, Half-Life, World of Warcraft, and Second Life. World of Warcraft, for example, has over 11.5 million users [3] with a peak of over 500,000 players online at a given time [1]. MMOGs have the potential to be the cornerstone of any eSociety platform in the near future, because they bring the massiveness, awareness, and inter-personal interaction of the real society into the digital realm.

Observations about the growth and evolution of MMOGs are confirmed by data from recent measurements. For the past few years, studies have concluded that these applications are expected to be major contributors to Internet traffic. DFC Intelligence forecasted the worldwide online game market to grow from $\$ 4.5$ billion in 2006 to over $\$ 13$ billion in 2011 and the total number of online gamers in the twenty leading online game countries is

\footnotetext{
${ }^{1}$ At the time of writing of this article, $1 \mathrm{US} \$=266.50 \mathrm{~L} \$$, with roughly L\$ 100 Million exchanged daily.

S. Shirmohammadi $(\bowtie)$

Distributed and Collaborative Virtual Environments Research Laboratory (DISCOVER Lab),

University of Ottawa, Ottawa, Canada

e-mail: shervin@discover.uottawa.ca

M. Claypool

Interactive Media and Game Development, Worcester Polytechnic Institute, Worcester, MA, USA

e-mail: claypool@cs.wpi.edu
} 
expected to increase $51 \%$ in that period. A detailed analysis by In-Stat/MDR reports that roughly $9 \%$ of the traffic sent back and forth over the US backbone was due to online gaming in 2002, and this is forecasted to reach nearly $30 \%$ by 2008 [2]. More recently, the NPD Group reports that $59 \%$ of total US population (ages 2 years old and higher) play games, with $56 \%$ of them doing so online, leading to almost $1 / 3 \mathrm{rd}$ of the population playing online games [4]. The former figure increases to $72 \%$, when considering the age group 6 to 44 years old. All reports and forecasts unanimously predict that these numbers will further increase over the next few years. As these online games become more popular and significant contributors to Internet traffic, research needs to be devoted to manage and support the emerging massiveness and its traffic on the network.

It is therefore not surprising that one of the main challenges in MMOGs is the issue of scalable networking and efficient traffic management. In fact, the first group of papers in this special issue deals with Networking Support and Client-server Optimization. To cover this important topic, the issue opens with the paper "On the Challenge and Design of Transport Protocols for MMORPGs", in which the authors evaluate TCP, UDP, DCCP, and SCTP in terms of their suitability for MMORPGs, and also propose novel transport strategies for this genre of games. "Improving SCTP Retransmission Delays for TimeDependent Thin Streams" follows the same line of thought but focuses more deeply on SCTP and its usage in MMOGs and similar applications, and proposes sender-side enhancements in SCTP that reduce latency in a manner that is compatible with unmodified receivers. "Using Geometrical Routing for Overlay Networking in MMOGs" proposes a new networking architecture based on geographical routing borrowed from wireless networks, and applies it with locality sensitive hashing to wired MMOGs. The next paper, "The Partial Migration of Game State and Dynamic Server Selection to Reduce Latency", uses a distributed name server for migrating objects between servers while ensuring that not only references to the moved objects are maintained, but also the aggregate latency for the affected players are decreased due to algorithm's locating an optimal server. Finally, this group of papers is finished off by "ALVIC-NG: State Management and Immersive Communication for Massively Multiplayer Online Games and Communities" which presents an extendable client-server architecture that allows for dynamic allocation of server capacity, combined with the ability to manage and moderate player activity.

The next topic is Player Interactivity and Traffic/Pattern Analysis Based on Human Behaviour. This topic opens with "On the Impacts of Human Interactions in MMORPG Traffic", where authors introduce a behavior detection algorithm to grab specific events and states from passive traffic measurements, make measurements using this algorithm, and introduce a technique for better traffic classification not considered in current methods. "Avatar Mobility in User-Created Networked Virtual Worlds: Measurements, Analysis, and Implications" collects mobility traces of avatars spanning multiple regions in Second Life, and analyzes the traces to characterize the dynamics of the avatars' mobility and behaviors, with implications on the design of future MMOGS. The next paper, "MMORPG Player Actions: Network Performance, Session Patterns and Latency Requirements Analysis", uses World of Warcraft as a case study to categorize five groups of player actions from the perspective of network traffic parameters, session characteristics, and latency, and discusses whether player activities may be suitable for implementation in a wireless handheld/mobile phone version. "The H-N2N Framework: Towards Providing Interperception in Massive Applications" proposes a framework designed and implemented for collaborative interaction of massive number of users through the Web by introducing the concept of interperception. Finally, "A DR Algorithm Based on Artificial Potential 
Field Method", introduces a new Dead Reckoning (DR) algorithm that creates a motion model among player entities based on artificial potential field and uses the weighted sum of certain parameters to make the predictive model better conform to the players' motions

The last group of papers deals with Zoning, Communities, and Security. Concerned with overloaded servers in certain "hot spot" zones due to higher-than expected player presence, "A Load Balancing Scheme for Massively Multiplayer Online Games" proposes a balancing scheme which allocates load on the servers proportionally to the each server's power while reducing as much as possible the overhead from the distribution. Citing high maintenance costs of the famous Delaunay Triangulation connectivity substrate, "Efficient Triangulation for P2P Networked Virtual Environments" searches for more practical alternatives and proposes a new variant to the Delaunay Triangulation algorithm that provides network connectivity to support P2P MMOGs while dramatically decreasing maintenance overhead. "A Hybrid P2P Communications Architecture for Zonal MMOGs" uses a graph-theoretic framework for $\mathrm{P} 2 \mathrm{P}$ overlay stabilization within a zone, further improved by interest-driven zone crossing, dynamic shared regions, and clustering of entities based on their attributes. Two papers finish off this special issue in communities and security, respectively. First, "Virtual Context Based Services for Multiplayer Online Games to Facilitate Community Participation" introduces the concept of virtual contextbased services to enable information exchange in a game and genre independent manner, and proposes a standardized way to describe the virtual context of a gamer that can be used to interface between games and other internet applications. Then, "Secure Peer-toPeer 3D Streaming" identifies practical security concerns and obstacles in P2P-based MMOGs and presents basic solutions and their respective security analysis to address these obstacles.

At the end, we would like to express our gratitude to the reviewers whose feedback and rigorous observations and comments directly influenced the high quality of the papers in these special issues. Without them, this work would not have been possible. Hence, we sincerely thank:

Anees Shaikh

Behnoosh Hariri

Carsten Griwodz

Christoph Rensing

David Finkel

Dewan Tanvir Ahmed

Gregor Schiele

Grenville Armitage

Jauvane C. de Oliveira

Marc Liberatore

Md Anwar Hossain

Mohsen Ghaffari

Mojtaba Hosseini

Pål Halvorsen

Shun-Yun $\mathrm{Hu}$

Surendar Chandra

Tobias Fritsch

Travis Schluessler

Wei Tsang Ooi
IBM T.J. Watson Research Center, U.S.A.

Sharif University of Technology, Iran

University of Oslo, Norway

Technische Universität Darmstadt, Germany

Worcester Polytechnic Institute, U.S.A.

University of Ottawa, Canada

University of Mannheim, Germany

Swinburne University of Technology, Australia

National Laboratory of Scientific Computing (LNCC), Brazil

University of Massachusetts, U.S.A.

University of Ottawa, Canada

Sharif University of Technology, Iran

Magor Communications Corporation, Canada

University of Oslo, Norway

National Central University, Taiwan

University of Notre Dame, U.S.A.

Freie Universität Berlin, Germany

Intel Corporation, U.S.A.

National University of Singapore, Singapore 


\section{References}

1. Edwards C (2006) "Hi, I'm the Prince of Azeroth. Let's Network”, Business Week, August 21 2006, http:// www.businessweek.com/magazine/content/06_34/b3998418.htm

2. Mantion E (2004) "Online Gaming: Where the Lost Boys Are”, In-Stat Research Report IN0401178IA, May 2004.

3. Wilson H (2008)“World of Warcraft Subscribers Now 11.5 Million", International Business Times, December 23 2008, http://www.ibtimes.com/articles/20081223/world-warcraft.htm

4. NPD Group (2008) “Online Gaming 2008”, http://www.npd.com

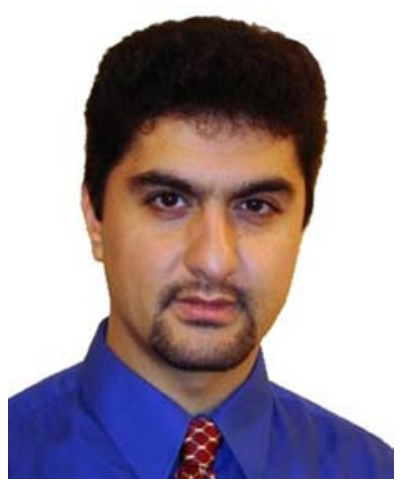

Shervin Shirmohammadi an IEEE Distinguished Lecturer, is Associate Professor at the School of Information Technology and Engineering, University of Ottawa, Canada. His current research interests include Massively Multiplayer Online Gaming (MMOG) and Virtual Environments, Application Layer Multicasting and Overlay Networks, Adaptive P2P Audio/Video Streaming, and Multimedia Assisted Rehabilitation Engineering. The results of his research have lead to more than 130 publications, over a dozen technology transfers to the private sector, and a number of awards and prizes. He is Editor-in-Chief of the International Journal of Advanced Media and Communications, Associate Editor of ACM Transactions on Multimedia Computing, Communications, and Applications, Associate Editor of Springer's Journal of Multimedia Tools and Applications, and also chairs or serves on the program committee of a number of conferences in multimedia, virtual environments, games, and medical applications. Dr. Shirmohammadi is a University of Ottawa Gold Medalist, a licensed Professional Engineer in Ontario, a Senior Member of the IEEE, and a Professional Member of the ACM. 


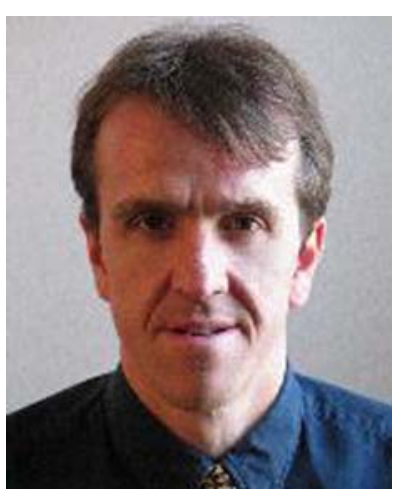

Mark Claypool is a Professor in Computer Science at Worcester Polytechnic Institute in Massachusetts, USA. He is also the Director of the Interactive Media and Game Development major at WPI, a 4-year degree in the principles of interactive applications and computer-based game development. Dr. Claypool earned M.S. and Ph.D. degrees in Computer Science from the University of Minnesota in 1993 and 1997 , respectively. His primary research interests include multimedia networking, congestion control, and network games. 